\title{
EL RESENTIMIENTO COMO ARGUMENTO MORAL ${ }^{15}$
}

En su conocida conferencia Freedom and Resentment, Peter Frederick Strawson se propone corregir el determinismo moral, tomando como punto de apoyo el resentimiento, es decir, la reacción natural que experimentamos cuando alguien nos causa una ofensa o una injusticia. Resulta interesante confrontar su propuesta con la doctrina moral de Spinoza, ya que ésta no solamente sostiene un fuerte determinismo, sino que, por el contrario, busca desarraigar del sabio el resentimiento, ya que éste produce tristeza, es decir, debilitamiento de las fuerzas morales, y debe ser contrarrestado con una adecuada comprensión de la causa que nos lo produce.

Para desarrollar mejor la confrontación, voy a comenzar exponiendo, en sus líneas fundamentales, la propuesta de Strawson, buscando determinar la estructura básica de su argumentación, para contrastarla luego con la doctrina de Baruch Spinoza.

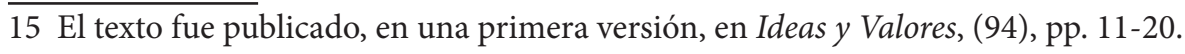




\section{La propuesta de Strawson}

Comencemos recordando que, en su artículo, Strawson distingue al menos tres grandes grupos de actitudes o sentimientos reactivos frente al comportamiento ajeno o propio. El primer grupo de actitudes reactivas podemos recogerlo bajo la pareja gratitud o resentimiento. Corresponde a la reacción que experimentamos cuando alguien nos trata justa y amablemente o, por el contrario, nos ofende. A tales actitudes reactivas podemos llamarlas personales, en cuanto nos hallamos directamente involucrados. Un segundo grupo comprende actitudes o sentimientos reactivos en los cuales no nos hallamos directamente involucrados; es el caso cuando vemos que alguien ofende o trata injustamente a un tercero. Estos sentimientos podemos resumirlos bajo el término general de indignación. Y un tercer grupo de actitudes reactivas se refiere a mi propio mal comportamiento con respecto a los demás, y lo podemos llamar vergüenza.

Vamos a fijar nuestra atención, como lo hace Strawson, en el primer grupo, es decir, en el resentimiento, porque allí se centra su argumentación, sin olvidar los otros sentimientos que conforman en su conjunto el tejido que fundamenta su propuesta moral. Podríamos resumir así su argumentación: el resentimiento es una actitud natural que exige explicación. Es verdad que puede ser neutralizada de dos maneras diferentes. Una es cuando caigo en la cuenta, o se me hace caer en la cuenta, de que el sujeto que causó la ofensa carece de responsabilidad moral: es un loco o un niño, para decirlo en pocas palabras. En este caso, el sujeto en cuestión está situado fuera de una relación verdaderamente interpersonal, ya que no se puede apelar a su capacidad de responder por sus actos. Una segunda forma de neutralizar el resentimiento consiste en señalar una disminución de la responsabilidad del ofensor: "estaba sometido a una gran presión" o "tiene un carácter irascible". Aquí el resentimiento no se espera que desaparezca, sino que se aminore, precisamente porque la relación interpersonal no se ha roto por completo, sino que se halla disminuida.

En esa forma, Strawson ha mostrado cómo el resentimiento (y las demás actitudes reactivas) se fundamentan sobre las relaciones interpersonales, es decir, consideramos a los demás tan responsables de sus actos como nos consideramos a nosotros mismos, y por ello nos resentimos de su trato 
injusto con nosotros (o de unos con otros, o de nosotros frente a ellos). Con lo cual cree Strawson estar en condiciones de tomar partido frente a las doctrinas contrapuestas del determinismo y del no determinismo. Confiesa, sí, no haber entendido nunca bien la doctrina determinista, y aunque considera que el indeterminismo va muy lejos en sus pretensiones metafísicas al no explicar los sucesos "tal como nosotros los entendemos", se comprende muy bien que rechace el determinismo por las consecuencias que éste acarrea. Busca entonces un camino intermedio que, sin caer en un indeterminismo incomprensible, le permita evitar las consecuencias indeseables del determinismo. Pero, ¿cuáles son esas consecuencias?

Consideremos para ello una posible actitud alternativa frente al resentimiento: ver al ofensor como alguien que obedece a una serie de determinaciones, de modo que su comportamiento se debe atribuir a ellas; en esta forma podemos comprender mejor su acción y buscar los medios más adecuados para influir sobre ella. Esta actitud objetiva o "científica" es precisamente la que debemos asumir frente a los ofensores con quienes no podemos establecer verdaderas relaciones interpersonales (locos o niños); pero ¿debemos también tomarla con los demás ofensores? Sin duda que en ocasiones particulares esta actitud objetiva u objetivante puede ser muy recomendable, pero la cuestión es: ¿puede ser generalizada?

Aquí está, precisamente, la consecuencia indeseable de un pretendido determinismo universal; porque nuestro resentimiento (y las otras actitudes reactivas) tendrían que desaparecer para dar lugar a actitudes objetivantes, con las cuales buscamos entender los móviles de un determinado comportamiento humano, así como la mejor manera de incidir sobre él para nuestro beneficio. Con ello, considera Strawson, las relaciones interpersonales se anularían, y entraríamos en un mundo donde las prácticas de condena moral o de castigo "tienen tan solo el carácter de instrumentos tácticos, de métodos de tratamiento individual y de control social" $\left(1974\right.$, p. 61/76) ${ }^{16}$. Un mundo así produce horror, porque en él se excluyen no solamente las actitudes reactivas morales, sino que también se "excluyen al mismo tiempo elementos esenciales de los concepto de condena moral y de responsabilidad moral" (1974, p. 63/76). Ahora

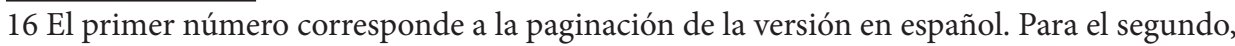
se da la paginación del texto tal como fue editado por Watson, por ser la fuente más común de citación en inglés, tal como lo indica la edición de McKenna, en la que me apoyo, y la cual indica esa misma paginación entre paréntesis cuadrados (ver Bibliografía: McKenna). 
bien, si las consecuencias inaceptables (e imposibles) del determinismo lo desautorizan como doctrina práctica, no por ello podemos caer en el indeterminismo que resulta simplemente incomprensible. ¿Qué podría significar que los acontecimientos, o algunos de ellos, son indeterminados?

La solución de Strawson, como las soluciones del empirismo tradicional, consiste en proponer un entendimiento mediante renuncias de las dos partes en conflicto, lo cual termina no siendo satisfactorio para ninguna de ellas. Strawson mismo es consciente de ello y lo declara sin ambages: "Esta conferencia quiere ser un paso hacia la reconciliación; así que es probable que a todo el mundo le parezca fruto de una actitud obstinada" (1974, p. 38/59). Ahora bien, ¿cuál es su propuesta? Realmente no resulta nada clara, y uno se siente tentado a reproducir el párrafo final de su escrito para mostrarlo. Intentaré, sin embargo, resumirlo. Nos viene a recomendar que asumamos el determinismo, pero modificándolo radicalmente, ya que tiene razón en enfatizar la eficacia que tienen las actitudes morales (condena moral, resentimiento, castigo, etc.) para controlar las conductas humanas, pero se equivoca al olvidar su carácter de reacciones morales, de expresiones de nuestra naturaleza humana. El objeto mismo de nuestras actitudes reactivas pareciera no poderse entender, pero no podríamos descartarlo, so pena de llegar a consecuencias catastróficas: un mundo sin relaciones interpersonales.

\section{Análisis de la propuesta}

Comienzo por señalar que la debilidad más evidente de la propuesta de Strawson, tal como puede verse a la luz de Spinoza, es su desconocimiento o incomprensión del determinismo. Porque si bien es verdad que comienza confesando que no sabe en qué consista propiamente la tesis del determinismo, todo su artículo constituye un alegato en contra de las indeseables consecuencias de esa tesis, demostrando, de manera indirecta, que tiene una versión muy clara de la misma, aunque muy discutible. Así que lo primero que voy a hacer es tratar de precisar qué entiende Strawson por determinismo. 
En primer lugar, el determinismo es para él una doctrina que propone asumir, en lugar de nuestras actitudes reactivas, actitudes objetivantes, es decir, considerar a todos los actuantes, incluido el observador, como objetos que se mueven por determinaciones que les son ajenas y que, por lo tanto, carecen de responsabilidad moral, de culpa o de mérito, en otras palabras, como seres incapaces de responder por sus acciones. Descartadas en esta forma las actitudes morales, el juego de las relaciones humanas se guía en forma exclusiva por criterios de eficacia y de utilidad.

Cabría, sin embargo, dice Strawson, establecer algunas distinciones, aunque solo fueran de carácter hipotético. Podemos suponer un mundo en el cual alguien asume de manera consecuente el determinismo, pero únicamente con respecto a los demás; esa persona sentiría vergüenza por sus acciones injustas con los demás, pero no sentiría resentimiento cuando sufriera una ofensa, ya que los demás carecerían para él de responsabilidad moral; ni sentiría tampoco indignación frente a las ofensas de unos contra otros, por esa misma razón. Tendríamos así una especie de 'santo', muy cercano a la tontería, pero que podría, sin embargo, parecerse al 'sabio' del spinocismo: él solo se sabe libre frente al vulgo, al que considera incapaz de acceder a la libertad.

Podríamos también pensar un mundo en el cual alguien, por el contrario, creyera que solo él carece de responsabilidad moral, al estar determinado a obrar como obra, mientras que los demás sí serían culpables cuando obran de manera incorrecta. Este caso, nos dice Strawson, sería aún más aberrante que el anterior, y constituiría "un caso anormal de egocentrismo moral, una especie de solipsista moral" (1974, p. 55/ p. 72). En realidad, nos advierte el mismo Strawson, las diferentes actitudes reactivas "en tanto que capacidades o predisposiciones humanas, se / mantienen o se desmoronan a la par [sic]" (1974, pp. 58-59/74).

En esta forma, por el camino de consideraciones morales, se nos ha ofrecido una imagen del determinismo que constituye una mezcla de insensibilidad moral y de imposibilidad real. Nada extraño, entonces, que se asemeje a la imagen que se han hecho desde sus comienzos los adversarios de Spinoza, al considerar que su doctrina es una forma de fatalismo. En realidad, lo que se busca es atacar un punto crucial de la doctrina spinocista, a saber, su rechazo al concepto mismo de pecado. 
Porque Strawson busca afincarse en las actitudes reactivas (resentimiento, indignación y vergüenza) como sentimientos que afloran en nosotros cuando creemos que quien ha actuado de manera incorrecta lo ha hecho en virtud de su libre albedrío. En otras palabras, las tres actitudes reactivas tienen en común el reaccionar ante una acción que se considera incorrecta o injusta, pero, al tratarse de sentimientos o actitudes reactivas, suponen que el sujeto de la acción en cada caso ha obrado con entera libertad, en el sentido de ser culpable de lo ocurrido. No se trata de constatar sin más la incorrección de una acción ajena o propia, sino de resentirla como el efecto de una mala intención, de una voluntad aviesa. Esto significa que quien ha actuado podía haber obrado de otra manera si lo hubiera querido, pero lo ha hecho así "con plena advertencia y con pleno consentimiento", como decía el viejo catecismo católico, el famoso "Astete", al definir el 'pecado'. Por ello, cuando uno mismo o alguien más busca neutralizar esas reacciones, apunta precisamente a negar esa voluntariedad (en el caso de los locos o de los niños), o a disminuirla. Y en esa misma dirección apunta la argumentación de Strawson, a saber, a mostrar que el determinismo descarta por principio toda culpabilidad, al asumir una actitud meramente objetiva; lo cual implica romper toda relación interpersonal, porque solamente es 'persona' quien es o puede ser responsable de sus actos, y solamente es tratado como persona aquel a quien se considera responsable de sus actos.

Podemos, entonces, reformular la argumentación de Strawson de la siguiente manera: tenemos actitudes reactivas porque sentimos que alguien ha "pecado" contra nosotros (resentimiento), que alguien ha "pecado" contra un tercero (indignación), o que nosotros hemos "pecado" contra alguien (vergüenza). Sin duda, es posible que a veces convenga prescindir del carácter 'pecaminoso' de la acción y examinar los hechos de manera objetiva, es decir, sin tener en cuenta las malas intenciones de quien ha obrado de manera incorrecta. Pero pretender volver totalmente objetivas nuestras relaciones interpersonales, hasta el punto de negar la posibilidad misma del pecado, como lo hacen los deterministas (Spinoza), viene a ser una propuesta inaceptable. Por una parte, no resulta realista, ya que esos sentimientos nos son connaturales y por ello universales, y resulta tan evidente que se cometen con frecuencia pecados en el comportamiento 
humano, que una teoría metafísica como el determinismo no puede pretender neutralizar tales sentimientos. Pero, además, sería aberrante pretenderlo, ya que, si creemos que todos pueden pecar menos nosotros, nos convertimos en unos egoístas morales, como pudimos verlo antes; y si creemos que solo nosotros podemos pecar, nos convertimos en unos 'santos' muy cercanos a la tontería. Por último, si creemos que nadie puede pecar, nos hallamos en el reino de la fantasía, que es a la vez el reino del más terrible cinismo. Resulta fantasioso, por pretender creer que nadie puede obrar mal, y resulta cínico, porque considera que las sanciones morales y jurídicas no son más que mecanismos para manipular la conducta de las personas.

Como podemos ver, si Strawson hubiera utilizado el concepto de 'pecado', quitándole el carácter religioso que suele tener y entendiéndolo como aquella acción que, a sabiendas, ofende o causa daño a alguien de manera injusta, su argumentación hubiera podido ser mucho más clara ${ }^{17}$. Pero con ello también hubiera sido más clara la fragilidad de su argumentación, así como la inevitable oscuridad que comporta su propuesta de solución. Veámoslo.

\section{El problema del "pecado"}

Para abordar el problema del "pecado", debemos pasar a confrontar la tesis de Strawson con la doctrina determinista de Spinoza, para lo cual será necesario hacer algunas precisiones sobre la misma. Porque, en contra de lo que parece pensar Strawson, el determinismo, al menos tal como lo sostiene Spinoza, no es una doctrina oscura y difícil de explicar. Sin duda que su justificación metafísica y sus consecuencias filosóficas exigen emprender un largo y profundo esfuerzo especulativo. Pero, en los términos de la confrontación que nos ocupa, podemos muy bien reducir el determinismo a dos tesis complementarias, que no creo que sean demasiado complejas.

1.a Tesis: el determinismo spinocista implica la identificación del entendimiento humano con la voluntad: Voluntas et intellectus unum et

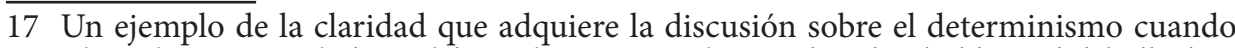
se utiliza el concepto de 'pecado' puede verse en el Tratado sobre la libertad del albedrío, de Anselmo de Canterbury y sobre todo, en el texto introductorio de Felipe Castañeda, titulado La libertad condicionada: un análisis del De libertate arbitrii de Anselmo de Canterbury (ver Bibliografía). 
idem sunt (la voluntad y el entendimiento son uno y lo mismo) [E2P49C]. Con ello se quiere decir que nuestra voluntad sigue siempre de manera necesaria los dictados del entendimiento, en el sentido de que cuando obramos, lo hacemos buscando aquello que el entendimiento nos dice que nos conviene más. En esta forma se entiende muy bien la operación de nuestra voluntad, ya que es movida por aquello que el entendimiento le muestra como apetecible.

A la objeción de que con frecuencia esto no parece ser cierto y que apetecemos lo que no nos conviene o aquello que no deberíamos hacer, la respuesta es simple: es cierto que podemos equivocarnos por no haber pensado de manera correcta, es decir, que podemos cometer errores (no pecados); errores que podemos llegar a corregir mediante una correcta reflexión. Spinoza no piensa que estemos exentos de error, por más cuidado que pongamos en el ejercicio de la reflexión, pero no ve en ello ninguna maldad. En otras ocasiones no se podrá hablar propiamente de error en sentido estricto, porque nuestra acción no ha sido el resultado de una correcta deliberación, sino que simplemente hemos seguido nuestros impulsos o nos hemos dejado arrastrar por nuestras pasiones. Esto también lo acepta Spinoza y lo tiene muy en cuenta, ya que le dedica toda la parte cuarta de su Ética a la "servidumbre humana", de la cual nos dice:

Llamo servidumbre humana a la impotencia para moderar y reprimir los afectos, porque el hombre sometido a los afectos no depende de sí, sino de la fortuna, bajo cuya potestad se encuentra de tal manera, que a menudo se ve compelido, aun viendo lo mejor, a hacer sin embargo lo peor (E4Prae).

Así que, según esta primera tesis del determinismo, cuando obramos es porque seguimos a nuestro entendimiento en la medida en que éste haya alcanzado ideas adecuadas, y cuando no seguimos a nuestro entendimiento, entonces propiamente no obramos, sino que padecemos, es decir, somos presa de nuestras afecciones. Sin embargo, en ninguno de los dos casos en que realizamos una acción incorrecta hay lugar para una mala intención, para una búsqueda intencionada del mal, ya que ello carecería de razón, sería simplemente inconcebible. Para hablar en términos clásicos, sería desear lo indeseable. 
Tal absurdo es, precisamente, lo que se pretende encubrir (o descubrir) con el término 'pecado'. De modo que la primera tesis del determinismo niega la posibilidad misma del pecado, al negar el concepto de un incomprensible 'libre albedrío', es decir, de una voluntad que desde sí misma y por sí misma escogiera precisamente aquello que el entendimiento le muestra como lo que no debe escoger ("con plena advertencia y con pleno consentimiento"). Toda teoría ética que en forma clara o velada pretenda mantener la idea de pecado o de libre albedrío como indispensable para salvaguardar el sentimiento moral, tiene que distinguir en alguna forma la voluntad del entendimiento; e inversamente, cuando una teoría moral defiende la autonomía de la voluntad frente al entendimiento, es porque tiene una visión pesimista del ser humano como alguien inmerso en el absurdo del pecado. Y esto último resulta interesante, porque, por lo general, se considera que cuando absolutizamos la voluntad estamos exaltando a los seres humanos hasta el nivel de la divinidad, como lo pensaba Descartes (4.a Meditación), pero en realidad los estamos poniendo por debajo de los animales, al hacerlos capaces de quebrantar la misma racionalidad, es decir, de comportarse peor que un ser irracional.

2.a Tesis: el determinismo considera que las acciones del ser humano se mueven entre dos formas de determinación, una externa, que proviene de los afectos (pasiones) y una interna, que proviene del entendimiento (acciones). Esta tesis complementa la anterior y es, en cierta forma, su consecuencia. Viene a ser propiamente la tesis del determinismo desde el punto de vista moral. Porque lo que se niega con ella es, de nuevo, que puedan darse acciones indeterminadas, acciones "porque sí", sin ninguna razón suficiente. Las acciones efectuadas por determinaciones externas son propiamente pasiones, y en ellas carecemos delibertad. Esas determinaciones externas pueden provenir de nuestras inclinaciones o 'pulsiones', como las llama el psicoanálisis, pero no por ello son menos externas a nuestra conciencia, es decir, no están bajo el dominio de nuestra voluntad. Y, en cuanto a las acciones que provienen de nuestra voluntad iluminada por el entendimiento, también son determinadas, es decir, tienen una razón, o sea, una determinación del entendimiento que nos señala aquello que debemos hacer, aquello que en verdad nos conviene. 
Considero que la defensa más lúcida y más consecuente que se ha hecho del libre albedrío se encuentra en la doctrina moral kantiana, y es en ella donde mejor se pueden apreciar los elementos incomprensibles a los que tal defensa nos conduce. De ahí que alguien familiarizado con esa doctrina podría objetarme que no resulta lícito identificar, como lo hace Spinoza, lo que debemos hacer y lo que más nos conviene. Pero esa identificación vale para alguien que, como Spinoza, confíe totalmente en la razón. Porque lo que debemos distinguir con claridad es aquello que parece convenirnos, pero que una razón ilustrada puede mostrar con claridad que no es el caso, de aquello que en realidad nos conviene, y que una razón ilustrada nos muestra como nuestro deber. Porque algo que debiéramos hacer, pero que no nos conviniera en ningún sentido racional del término, sería para Spinoza algo simplemente inconcebible, y una idea así se halla conectada con la de libre albedrío. No debemos confundir lo que nos dicta nuestra razón con lo que nos señalan nuestras inclinaciones y nuestros apetitos. Dicho en otras palabras, nuestros intereses particulares no son en realidad nuestros verdaderos intereses.

Ahora bien, al situar el comportamiento humano entre dos determinaciones, unas internas y otras externas, Spinoza se preocupa por señalar que en ambos casos obramos de manera natural: una veces seguimos los impulsos de la naturaleza y otras los intereses de nuestra naturaleza racional. De modo que, cuando buscamos con nuestro entendimiento los intereses de nuestra naturaleza racional, no quebrantamos las leyes de la naturaleza, sino que las reorientamos en nuestro propio beneficio, ya que la naturaleza como tal no se preocupa para nada por nuestros intereses. Pero lo que en todo caso resulta imposible es obrar en contra de los dictados de la razón "con plena advertencia y con pleno consentimiento".

\section{Consecuencias del determinismo}

Como nos indica Strawson con gran preocupación, el determinismo implica, en primer lugar, que la actitud racional frente a las ofensas que los demás nos infligen, o que las personas se infligen unas a otras, o que nosotros infligimos a los demás, debería ser una actitud objetivante y no reactiva, es decir, abandonar el resentimiento, la indignación o la vergüenza, 
para buscar serenamente las motivaciones de tales acciones y aplicar los correctivos correspondientes. Pero, de nuevo según Strawson, esa actitud objetivante no puede ni debe generalizarse. No puede, porque una teoría general como el determinismo es incapaz de neutralizar sentimientos tan naturales y tan universales como ésos que tenemos cuando se produce una ofensa o una injusticia. Y tampoco debe generalizarse, porque ello conduciría a romper las relaciones interpersonales y a tratarnos unos a otros como simples objetos de manipulación recíproca.

Sin embargo, el determinismo de Spinoza, tal como lo he presentado, no acarrea en verdad las consecuencias que Strawson teme. En primer lugar, porque no cabe esperar que todos o la mayoría de los seres humanos lleguen a adoptarlo, ya que para llegar a comprenderlo de tal manera que se constituya en una actitud vital, es necesario un esfuerzo especulativo muy arduo. Recordemos lo que nos dice Spinoza al terminar su Ética:

El sabio, en cuanto se lo considera como tal, difícilmente se conmueve en su ánimo, sino que, consciente de sí, de Dios y de las cosas con cierta necesidad eterna, nunca deja de ser, sino que siempre posee la verdadera satisfacción del ánimo. Pero si el camino que he demostrado que conduce a este fin parece arduo, sin embargo es posible hallarlo. Y ciertamente debe ser arduo lo que se encuentra tan raramente. ¿Cómo, en efecto, sería posible, si la salvación estuviera al alcance de la mano y si pudiera conseguirse sin gran esfuerzo, que la descuidaran casi todos? Ahora bien, todo lo excelso es tan difícil como raro. (E5P42S).

En segundo lugar, frente a lo que llamamos un ofensa, el sabio tiene dos opciones claras y racionales: si el ofensor tiene capacidad de reflexionar, deberá tratar de conducirlo a ello para que recapacite y cambie de actitud al corregir su comportamiento, porque se trata de un error o de una debilidad, no de una maldad; pero si el ofensor es incapaz de reflexión o se niega ello, no es el sabio quien rompe la relación interpersonal, como pareciera creerlo Strawson, sino que simplemente constata que esa relación ha sido rota por parte del ofensor. Pero tampoco entonces tiene cabida el resentimiento o la indignación, porque no se ha configurado un "pecado" o una maligna culpabilidad. Lo razonable es acudir a los mecanismos disponibles para reorientar la acción de quien obra sin guiarse por su razón. Y entre esos mecanismos está el suscitar resentimiento o indignación, acudir a la justicia 
y al castigo, tomar precauciones o acudir a la propia defensa. Y en cuanto a la vergüenza que podamos sentir por nuestras acciones incorrectas, ella solo tiene sentido como reconocimiento de nuestro error o nuestra debilidad, y debe llevarnos a buscar correctivos, pero no propiamente al remordimiento, que como tal nada aporta para cambiar nuestra forma de comportarnos: "El arrepentimiento no es virtud -dice Spinoza-, o sea que no nace de la razón; sino que quien se arrepiente de lo hecho es doblemente miserable o impotente" (E4P54).

En tercer lugar, el sabio comprende muy bien que las actitudes que debe esperar de sí mismo como resultado de un gran esfuerzo de reflexión no pueden ser las mismas que se pueden esperar de quien no haya recorrido ese arduo camino. Los sentimientos reactivos (resentimiento, indignación, vergüenza) son así mecanismos parcialmente efectivos para evitar que el ignorante -quien "aparte de ser zarandeado de múltiples maneras por causas exteriores y no gozar nunca de la verdadera tranquilidad, vive además como inconsciente de sí mismo y de Dios y de las cosas" (E5P42S)venga a irrumpir peligrosamente en las vidas ajenas. A estos sentimientos cabe añadir todos los demás mecanismos sociales y jurídicos (derecho, justicia, religión, etc.), mediante los cuales se logra reorientar las acciones de los particulares en beneficio de la sociedad.

Por último, cabe señalar que, al liberarnos de sentimientos tan negativos como el resentimiento, la indignación y la vergüenza, no solo logramos una paz y tranquilidad muy positivas, sino que, lejos de despreciar a nuestros semejantes o de romper nuestras relaciones interpersonales, como lo teme Strawson, aprendemos más bien a relacionarnos con ellos en la medida en que nos lo permita la razón. Considerar que el mundo está lleno de ignorancia, y que nuestra lucha por mejorarlo consiste en buscar superar esa ignorancia en nosotros mismos y en los demás, viene a conformar una actitud más interpersonal que pensar que está lleno de pecado y de maldad, y alimentar así el resentimiento, la indignación o la vergüenza.

En realidad, Strawson entiende el determinismo como la tesis según la cual todo está determinado desde fuera por causas que le son ajenas, y ante ello solo nos quedaría la opción del indeterminismo, es decir, la de que algo al menos sucede sin causa; y entre esos dos extremos, igualmente inaceptables, busca un camino intermedio impracticable. Pero la verdad del determinismo, 
en la versión de Spinoza, es que todo está determinado, ya sea por una causa externa, ya sea por una interna, y que nada puede ser indeterminado, porque sería pretender que algo ocurra sin una causa. Así que solo tenemos en verdad dos opciones: dejarnos determinar por causas externas o asumir con nuestro entendimiento la determinación de nuestra conducta, es decir, no ser libres o conquistar nuestra libertad. De esa manera, cuando alguien nos ofende ( $\mathrm{si}$ la ofensa es realmente tal y no un falso resentimiento de parte nuestra), se debe a que el ofensor no ha obrado de acuerdo con la razón, es decir, que ha sido determinado total o parcialmente por causas externas a su conciencia. Si vemos que es posible hacerlo reflexionar para que reasuma plenamente el control de su comportamiento, debemos apelar al diálogo y al razonamiento; pero, en la medida en que ello no resulte posible, tendremos que acudir a los mecanismos disponibles para reorientar esa conducta y evitar los daños que pueda causar.

Ahora bien, antes de terminar estas consideraciones, quiero responder a una maligna observación de Strawson contra el determinismo y contra la propuesta de asumir actitudes objetivantes. Si debemos tratar a todos los ofensores de manera objetiva, ¿por qué, se pregunta Strawson, no hacerlo así con todos los seres humanos? Y luego añade: "Más aún, ¿a quién podría, en un sentido general, hacerse esta recomendación? Únicamente a los poderosos, a las autoridades. Y con ello los abismos parecen abrirse" (1974, p. 62/77).

Si entiendo correctamente la sugerencia, Strawson señala el peligro de liberar a las autoridades de todo sentimiento de culpa. Sería como entregarnos a la cínica manipulación del poder por quienes poseen los medios para condicionar el comportamiento de los ciudadanos. Parece estar viendo, detrás del determinismo, la figura amenazante del Estado totalitario. Si es así, ello no pasaría de ser una mera fantasmagoría, fruto de una pobre comprensión del determinismo como si se tratara de una doctrina que negara toda autodeterminación, es decir, toda posible libertad.

En realidad, uno de los propósitos del determinismo, comprendido de manera adecuada, es descargar al Estado de la imposible tarea de sustentar juicios morales, para que pueda así desempeñar sus labores con la objetividad que le corresponde. Su función es dar cumplimiento a las reglas que la sociedad haya establecido libremente para su convivencia. También puede 
y debe distinguir entre quienes son capaces de ser llamados a la reflexión y quienes no pueden serlo. Por eso se habla de las prisiones como lugares para resocialización de los reclusos. Pero el castigo que impone el Estado no responde a una presunta responsabilidad moral del acusado, que los jueces no están en condiciones de establecer. De internis neque Ecclesia iudicat (de lo interior no juzga ni siquiera la Iglesia), decía sabiamente un viejo adagio; mucho menos podrá hacerlo el Estado. Podríamos, entonces, devolver la maligna advertencia de Strawson y preguntarnos si esos regímenes totalitarios a los que tanto teme, con sobrada razón, no han sido más bien el resultado de "sentimientos reactivos" desbordados: el resentimiento de un pueblo humillado, la indignación ante un proletariado sometido a la explotación y la vergüenza de quienes han cometido esos atropellos. 